\title{
SIMULATION AND EXPRESSION OF ATMOSPHERIC POLLUTION DISPERSION PROCESS BASED ON 3D GRID
}

\author{
Jiasong Shi ${ }^{1}$, Xiaochong Tong ${ }^{2}$, Weiming Yang ${ }^{3}$, Yunfen Chang ${ }^{1}$, Yan Zhong ${ }^{1}$, Xijie Gai ${ }^{1}$, Qinwen Zuo ${ }^{1, *}$ \\ ${ }^{1}$ State Key Laboratory of NBC Protection for Civilian, Beijing, China - qwz0228@gmail.com \\ ${ }^{2}$ Institute of Surveying and Mapping, Information Engineering University,Zhengzhou,China - txchr@aliyun.com \\ ${ }^{3}$ Academy of System Engineering, Beijing, China - damoyc@163.com
}

KEY WORDS: 3D Grid, Atmospheric Diffusion, Cellular Automata, Data Model, Simulation

\begin{abstract}
:
Aiming at the problems existing on the expression of atmospheric pollution dispersion, a data model of state-oriented and objectoriented hybrid modeling is designed. The model supports true three-dimensional grid representation and cellular automata deduction, and can combine the calculation and deduction of atmospheric pollution to improve the accuracy and efficiency of the model. The simulation experiments of single source and multi-source pollutants are carried out. The results show that: the model conforms to the diffusion law of atmospheric pollution. The pollutant concentration at any time, section or space in the whole diffusion process can be calculated and expressed dynamically, which improves the accuracy, rapidity, intuitiveness and analysation of the deduction of atmospheric pollutant diffusion process.
\end{abstract}

\section{INTRODUCTION}

At present, the derivation of the diffusion process of atmospheric pollution is based on the theory of atmospheric diffusion. By describing the diffusion and transportation processes of pollutants, the distribution of atmospheric pollution over a period of time is calculated to realize the assessment and prediction of atmospheric pollution. The atmospheric diffusion simulation process of atmospheric pollution consists of data preparation, diffusion modeling calculation, and diffusion process deduction expression. Commonly used atmospheric diffusion models include Gaussian models (Raza et al., 2005), Lagrangian puff models (Thykier-Nielsen et al., 1999; Yu et al., 2000), Lagrangian random walk models (Yan et al., 2009; Zhang et al., 2009), Euler models (Lauritzen et al., 2006; lagzi et al., 2004), and CFD turbulence models (de Sampaio et al., 2008). These atmospheric diffusion models are relatively mature, but the expression of the diffusion process is very inadequate (Xu et al., 2009).

The visualization of atmospheric diffusion mainly uses geographic information system (Wang et al., 2011; Sun et al., 2011; Chen et al., 2011; Zhang et al., 2010; Vienneau et al., 2009; Theophanides et al., 2009). Elbir (Elbir, 2004) uses the integration of geographic information system and Lagrange smoke model to estimate the spatial distribution and emission characteristics of pollutant air diffusion. Li (Li et al., 2007) realizes the visualization of harmful atmospheric diffusion based on the integration of standard Gaussian plume model and geographic information. $\mathrm{Hu}(\mathrm{Hu}$ et al., 2013) used cube to express air pollution, and connected six adjacent calculation points with six faces to understand the overall spatial distribution of air pollutants. Chen (Chen et al., 2013) constructs the geometric spatial position of each entity in the scene according to the actual three-dimensional geographical coordinates, presents the whole process of diffusion in the quasi three-dimensional scene, and dynamically predicts and displays the three-dimensional concentration of diffusion in real time. Although the above methods can present spatial distribution state, they all have information loss and cannot provide true three-dimensional expression. In addition, due to the separation of calculation and expression, the data need to be converted and resampled many times, resulting in accuracy reduction and efficiency loss.

In order to solve the shortcomings of the traditional methods, a new expression model of atmospheric pollutant diffusion based on the three-dimensional grid will be studied from the perspective of data model, and the diffusion process will be effectively expressed by the way of three-dimensional cellular automata.

\section{RESEARCH ON DATA MODEL IN THE PROCESS OF POLLUTANT DIFFUSION}

\subsection{Analysis of existing spatial data model}

At present, there are three kinds of spatial data models: 2D spatial data model, quasi 3D spatial data model and true 3D data model:

(1)The two-dimensional spatial data model is used to describe the spatial objects on the earth's surface. It can be expressed as $F=f(L, B)$, where $L$ and $B$ are the longitude and latitude of the location of the space object, $F$ is the attributes of the space object, and $f$ is the mapping relationship between the space location and the attributes.

(2)The quasi-3D spatial data model can be divided into $2.5 \mathrm{D}$ spatial data model and $2.75 \mathrm{D}$ spatial data model. In the $2.5 \mathrm{D}$ spatial data model, elevation is a continuous function of two independent variables of $L$ and $B$. It can be expressed as $Z=g(L, B) .2 .75 \mathrm{D}$ spatial data model can be expressed as $F=f(L, B, H), H=h_{1}, h_{2} \ldots h_{n}$. 2.75D spatial data is based on latitude and longitude, and most of the height information is lost. (3) In the real 3D spatial data model, the internal and surface attributes of spatial objects can be stored, which is a spatial body model. In the real 3D modeling process, $(L, B, H)$ are three independent parameters, which can be expressed as: $F=f(L, B, H)$. This model provides the possibility for accurate observation and understanding of the real world (Zhu et al., 2004).

The diffusion of gaseous pollutants is a typical threedimensional problem, so it is necessary to choose a proper three-dimensional model. In addition, there are two different 
types of spatial data model, one is object-oriented data model, the other is spatial state oriented data model. This paper analyzes the two types of data model and their application in the process of air pollutant diffusion expression.

\subsection{True 3D expression and cellular model based on 3D grid}

In order to organize, calculate and express the data of spatial field of air pollutants, a true three-dimensional spatial data model is needed.

In this paper, regular hexahedron is used as the true threedimensional model. Octree model is used for regular hexahedron subdivision. The octree model divides a cube into eight small cubes repeatedly, as shown in Figure 1 (a). As long as they are uneven inside, the individual cubes will be subdivided until they are even. In this way, the original data field becomes a regular arrangement of cubes with different sizes. The octree structure can be regarded as a variable block model. Simple areas are represented by large blocks, while complex areas are represented by small blocks.

For data field represented by $2 n \times 2 n \times 2 n$ grid stored in octree structure, the three-dimensional coordinates of a cell are $(I, J$, $K),(I, J, K=0,1,2,2 n-1)$, as shown in Figure 1 (b).Considering the different diffusion range and diffusion speed of pollutants, the coding method of octree needs to adopt the multi-scale coding method. In the process of selecting grid coding, the multi-scale integer coding $C$ proposed in (Lai et al., 2018) can save storage space and complete the calculation quickly. The transformation relationship between grid coding $C$ and coordinates $(I, J, K)$ can be quickly obtained by the method in (Lai et al., 2018). Therefore, the essence of coding $C$ is a multiscale way of three-dimensional Mordon code (Orenstein et al., 1984). The transformation between grid coding $C$ and threedimensional coordinates can be directly completed in bit operation, which is very convenient.

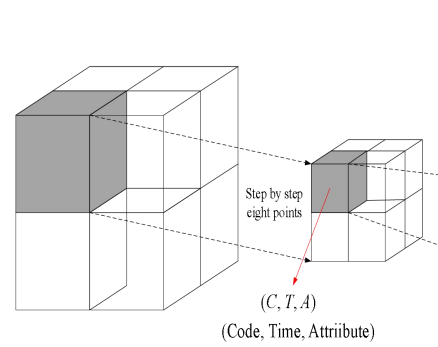

(a) Division method of $3 \mathrm{D}$ grid (Octree)

Figure 1. Division method and Coordinate recording method of $3 \mathrm{D}$ grid

Cellular model is a kind of mathematical model based on grid model, which is used for the evolution of group intelligence. It is often used in many fields, such as natural environment, social problems, scientific computing and so on, through the simple conversion rules between adjacent grid cells and the group computing of grid. Most of the common cellular applications are based on two-dimensional grid space. Although there are three-dimensional applications in water pollution, geological change and so on, there are few researches in the process simulation of atmospheric pollution.

In the expression of atmospheric pollutant diffusion process by using solid grid and cell model, the three tuple $(C, T, A)$ model is used to represent the spatial grid object, and the spatial position information $(C)$, state information $(A)$ and time information $(T)$ of the spatial object are related by grid code, as shown in Figure 1 (a).

\subsection{Mixed data model of object-oriented and state oriented}

The essence of the diffusion of atmospheric pollution is the field diffusion, and its spatial scope is not clear. The object-oriented spatial data model needs to describe the spatial range accurately and record the diffusion range with spatial coordinates, while the boundary of the field itself is fuzzy. Therefore, the objectoriented data model is not suitable for the deduction and expression of the diffusion process of atmospheric pollution. In the object-oriented data model, the object ID + time t of the is used as the identification of the object to record the state of the object at different times and realize the modeling of the dynamic spatial data, as shown in Table 1 .

On the basis of object-oriented data modeling, this paper designs a state oriented data modeling method, which emphasizes the description of the state and describes the state of each grid at a specific time, as shown in Table 2. As a typical space-oriented modeling method, compared with the traditional object-oriented modeling method, this method is more suitable for describing the dynamic changing field and the object with unclear boundary.

\begin{tabular}{|c|c|c|}
\hline Object ID & Time & State \\
\hline$A_{1}$ & $t_{0}$ & $s_{1}$ \\
$A_{1}$ & $t_{1}$ & $s_{2}$ \\
$A_{1}$ & $t_{2}$ & $s_{3}$ \\
$\ldots$ & & \\
$A_{2}$ & $t_{0}$ & $s_{4}$ \\
$A_{2}$ & $t_{1}$ & $s_{5}$ \\
$\ldots$ & & \\
\hline
\end{tabular}

Table 1. Object-oriented data modeling

\begin{tabular}{|c|c|l|}
\hline Code ID & Time & \multicolumn{1}{|c|}{ Attribute } \\
\hline$C_{1}$ & $t_{0}$ & $A_{2}, A_{4}, A_{7}, A_{8} \ldots$ \\
$\ldots$ & $\ldots$ & $\ldots$ \\
$C_{N}$ & $t_{0}$ & $A_{3}, A_{6}, A_{9}, \ldots$ \\
$\ldots$ & $\ldots$ & $\ldots$ \\
$C_{1}$ & $t_{1}$ & $A_{2}, A_{8}, A_{9}, \ldots$ \\
$\ldots$ & $\ldots$ & $\ldots$ \\
$C_{N}$ & $t_{2}$ & $A_{4}, A_{6}, A_{8}, A_{10} \ldots$ \\
\hline
\end{tabular}

Table 2. Data modeling based on state information

In application, it is necessary to build a data model combining object-oriented and state oriented. First, we need to build an object-oriented data model. Because the cell itself has the evolution process, but its attribute information is static, which is not suitable for storage with dynamic information. Different types of cells and their related data can be stored separately, only need to ensure the relevance between them; Secondly, we need to build a state oriented data model to reverse manage the cell ID according to the state value at a certain time, so as to quickly index cell information through the state. This model is very suitable for cell expansion process query, as shown in Figure 2.

In a word, the mixed data model is used to obtain cell state. In the diffusion simulation, the state oriented data model is mainly used. At the same time, the dynamic information of cell data needs to be queried many times. Any factors that affect cell state change, such as environment change, need to use objectoriented query, and the attributes need to be interpolated or extrapolated according to the time of the object. Therefore, in 
the process of diffusion simulation, both kinds of data models are necessary.

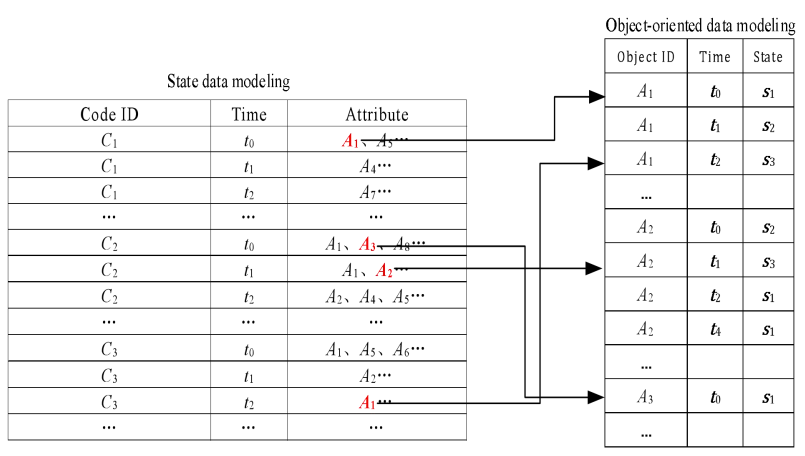

Figure 2. Mixed data model: mixed state modeling and object-oriented modeling

\section{SIMULATION OF ATMOSPHERIC DIFFUSION PROCESS BASED ON THREE-DIMENSIONAL GRID}

In this paper, the neighbor model of cellular automata is a threedimensional von Neumann neighbor model, which is adjacent to the central cell and neighbor cell surface. In the global solid grid, the neighbor direction of the central cell is defined as shown in Figure 1 (b). The direction of latitude increase is right, and the direction of latitude decrease is left; the direction of longitude increase is front, and the direction of longitude decrease is back; the sphere radius increase is up, and the sphere radius decrease is down. The neighbor cells in the three-dimensional grid are the cells in the left, right, front, back, up and down directions of the central cell.

\subsection{Evolution rule}

Evolution rule is the core of cellular automata, and it is the function that controls the dynamic evolution of cellular automata. In order to combine the atmospheric diffusion model of gaseous pollutants with the theory of cellular automata, the diffusion equation must be discretized. In this paper, the diffusion equation is discretized by using finite difference method. The basic idea is to discretize the diffusion region into a grid composed of grid points, and then the finite difference formula approximately replaces the derivative of the corresponding grid points. Finally, the approximate solution of the diffusion equation is obtained.

In order to improve the accuracy, the second-order central difference method in the finite difference method is selected in this paper. The specific form of the central difference model is shown in Figure 1 (b). $(i, j, k)$ represents the spatial position of a cell, corresponding to the $\mathrm{X}, \mathrm{Y}, \mathrm{Z}$ directions respectively; $\Delta x, \Delta y$, $\Delta z$ represents the distance between the cell and the adjacent cells in the $\mathrm{X}, \mathrm{Y}, \mathrm{Z}$ directions, which is equivalent to dividing the whole three-dimensional grid space into grid cells with side length of $\Delta x, \Delta y, \Delta z .(i+1, j, k),(i-1, j, k)$ represents $(i, j, k)$ adjacent grids in the front and back directions of $\mathrm{X}$ axis; $(i, j+1$, $k),(i, j-1, k)$ represents $(i, j, k)$ adjacent grids in the right and left directions of $\mathrm{Y}$ axis; $(i, j, k+1),(i, j, k-1)$ represents the adjacent grid of $(i, j, k)$ in the up and down directions of $Z$ axis.

The concentration function of atmospheric pollutant diffusion can be expressed in time and space as follows:

$$
C=C(x, y, z, t)
$$

In Formula $1, x, y, z$ is the variable of direction on threedimensional scale, and $t$ represents the variable of time. In the diffusion process of gaseous pollutants, the cellular automata rule of pollutant concentration field can be expressed as:

$$
C_{o}^{t+1}=f\left(C_{o}^{t}, C_{p}^{t}\right)
$$

In Formula 2, $C$ is the concentration value, $o$ is the central cell, $p$ is the neighbour cell, $t$ is the current time, and $t+1$ is the next time. It can be seen from the above formula that the state of the central cell at the next moment is determined by the current state of the central cell and the state of the neighbouring cells. Therefore, it is necessary to discretize the diffusion equation of pollutants into a form conforming to cellular automata. The diffusion equation of atmospheric pollution is as follows:

$$
\frac{\partial C}{\partial t}=-u \frac{\partial C}{\partial x}-v \frac{\partial C}{\partial y}-w \frac{\partial C}{\partial z}+\frac{\partial C}{\partial x}\left(K_{x} \frac{\partial C}{\partial x}\right)+\frac{\partial C}{\partial y}\left(K_{y} \frac{\partial C}{\partial y}\right)+\frac{\partial C}{\partial z}\left(K_{z} \frac{\partial C}{\partial z}\right)+S+D+R
$$

In Formula 3, S, D, R represents the source, dry and wet deposition and decay of the pollutant respectively. $u, v, w$ is the three components of wind speed in coordinate system, $K_{x}, K_{y}, K_{z}$ is diffusion parameter, $u \frac{\partial C}{\partial x}, v \frac{\partial C}{\partial y}, w \frac{\partial C}{\partial z}$ is used to describe the influence of wind on pollutant diffusion, $\frac{\partial C}{\partial x}\left(K_{x} \frac{\partial C}{\partial x}\right), \frac{\partial C}{\partial y}\left(K_{y} \frac{\partial C}{\partial y}\right), \frac{\partial C}{\partial z}\left(K_{z} \frac{\partial C}{\partial z}\right)$ is used to describe the influence of turbulence on pollutant diffusion.

After the second-order central difference of formula 3, formula 4 can be obtained:

$$
C_{i, j, k}^{t+1}=\left[\begin{array}{l}
-u\left(\frac{C_{i+1, j, k}^{t}-C_{i, j, k}^{t}}{\Delta x}\right)-v\left(\frac{C_{i, j+1, k}^{t}-C_{i, j, k}^{t}}{\Delta y}\right)-u\left(\frac{C_{i, j, k+1}^{t}-C_{i, j, k}^{t}}{\Delta x}\right) \\
+K_{x}\left(\frac{C_{1-1, j, k}^{t}+C_{i+1, j, k}^{t}-2 C_{i, j, k}^{t}}{\Delta^{2} x}\right)+K_{y}\left(\frac{C_{i, j-1, k}^{t}+C_{i, j+1, k}^{t}-2 C_{i, j, k}^{t}}{\Delta^{2} y}\right) \\
+K_{z}\left(\frac{C_{i, j, k-1}^{t}+C_{i, j, k+1}^{t}-2 C_{i, j, k}^{t}}{\Delta^{2} z}\right)+S_{i, j, k}^{t}+D_{i, j, k}^{t}+R_{i, j, k}^{t}
\end{array}\right] \cdot \Delta t+C_{i, j, k}^{t}
$$

$\Delta x, \Delta y, \Delta z$ is the spatial discrete scale, $\Delta t$ is the temporal discrete scale, $i, j, k$ is the sequence number of the spatial grid, $t$ is the time, and other parameters have the same meaning of formula 3 .

Formula 4 is the evolution rule of the cellular automata model of air pollutant diffusion, that is, the concentration value of the central cell at time $t+1$ is determined by the concentration value of the neighbor cell in six directions at time $t$ and the concentration value of the central cell at time $t$.

\subsection{Grid processing of boundary and emission source}

For the boundary grid, because there is no neighbor grid outside the boundary, the above evolution rules cannot be used directly. Virtual grid (blue grid in Figure 3) can be added outside the boundary grid (B in Figure 3), and the concentration gradient on both sides of the boundary grid is assumed to be the same, that is $\mathrm{C}_{\mathrm{B}}-\mathrm{C}_{\mathrm{B}-1}=\mathrm{C}_{\mathrm{B}+1}-\mathrm{C}_{\mathrm{B}}$.

Through this method, the concentration of the virtual grid is calculated, and then the next time concentration of the boundary grid is calculated by using the evolution rule.

For the grid where the emission source is located, in addition to the evolution simulation shown in formula (4), an increase in concentration shall be added before each iteration of the simulation. If the emission source is regarded as constant emission, the concentration increase value can be expressed as a parameter. 


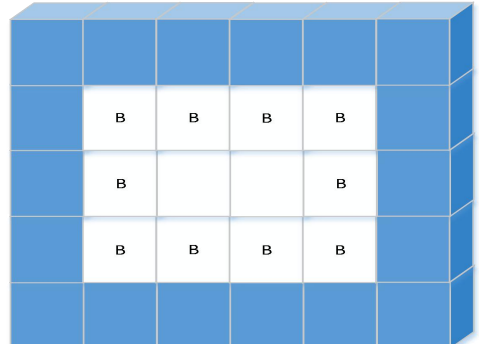

Figure 3. Boundary grid and virtual grid

\subsection{Operation process}

At the initial time, the concentration of the grid where the emission source is located is set to $\Delta t / V$, and the concentration of other grids is 0 . The weather of each grid needs to be known and can come from the real weather field. The diffusion parameters $\left(K_{x}, K_{y}, K_{z}\right)$ of each grid can be estimated according to the empirical model combined with the current meteorological conditions, and the estimation method is shown in reference.

According to the initial grid concentration state, meteorological parameters, diffusion parameters and evolution rules, the next grid concentration can be calculated. The calculation is repeated until the grid concentration tends to be stable.

\section{SIMULATION TEST}

Three experiments are designed to verify the data model and diffusion process. The first two experiments are atmospheric diffusion simulation experiments with a single release source and multiple release sources. The third experiment uses real wind field data. In the experiment, the pollutant concentration of each grid at different times are expressed.

\subsection{Simulation experiment}

\subsubsection{Experiment 1: Simulation of atmospheric diffusion of single emission source}

The range of diffusion simulation is $(0,0,0) \sim(500,500,500)$, the unit is meter, the discrete scale of space is $10 \mathrm{~m}$, the discrete scale of time is $0.1 \mathrm{~s}$, the release location is $(0,200,200)$, and the release source intensity is $20 \mathrm{~kg} / \mathrm{s}$. The wind speed in the whole diffusion area is constant. The wind speed in horizontal $u$ direction and $v$ direction is $5 \mathrm{~m} / \mathrm{s}$ and $2 \mathrm{~m} / \mathrm{s}$ respectively, and the wind speed in vertical direction $w$ is $0 \mathrm{~m} / \mathrm{s}$. The diffusion parameters in the direction are $10 \mathrm{~m}^{2} / \mathrm{s}, 10 \mathrm{~m}^{2} / \mathrm{s}$, and $2 \mathrm{~m}^{2} / \mathrm{s}$, Figure 4 shows the simulation results at different times.

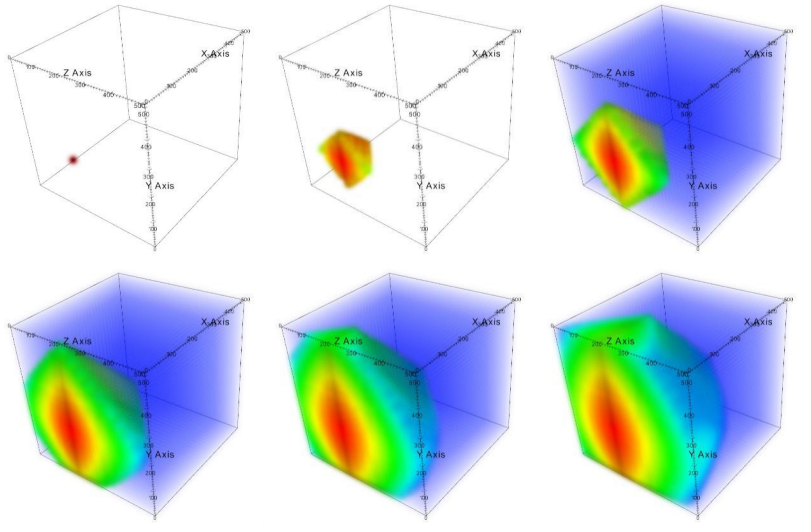

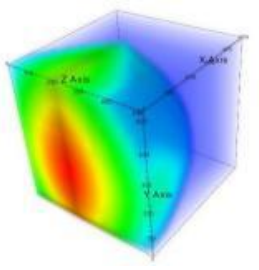
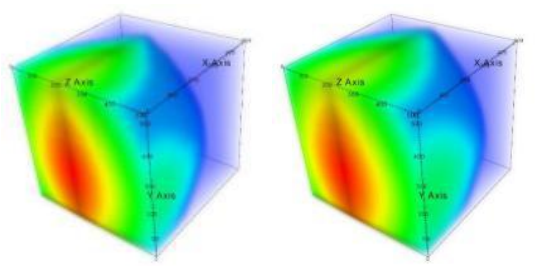

Figure 4.Simulation of experiment 1 (every 10 iterations)

\subsubsection{Experiment 2: Atmospheric diffusion simulation of multiple emission sources}

Except for the different release sources, the other conditions are the same as experiment 1 . The release positions of the two sources are $(0,150,150)$ and $(0,350,350)$, and the source strengths are $20 \mathrm{~kg} / \mathrm{s}$ and $10 \mathrm{~kg} / \mathrm{s}$ respectively. Figure 5 shows the simulation results of this experiment.
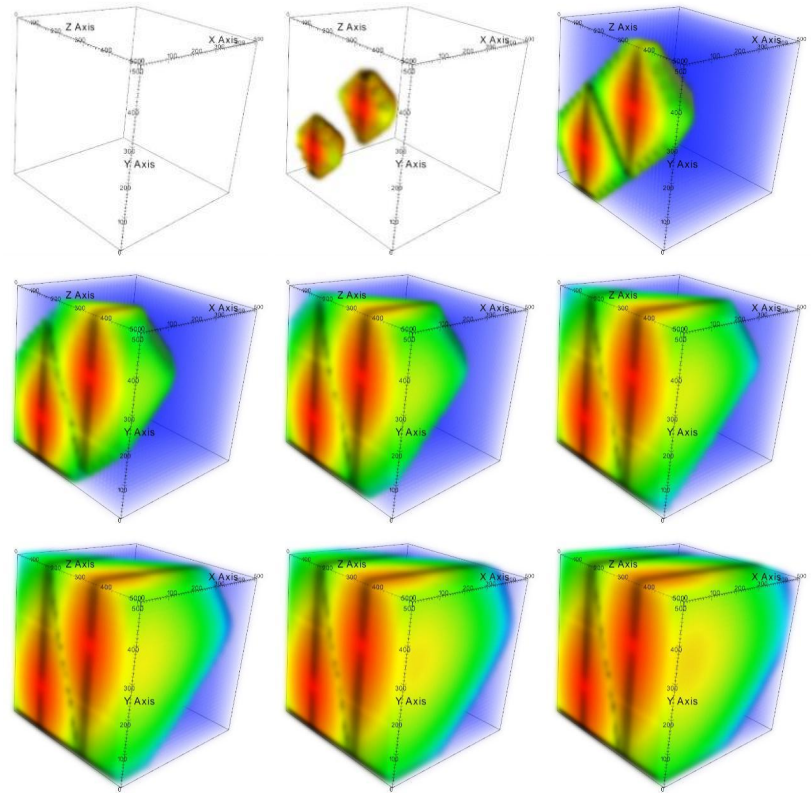

Figure 5. Simulation results of experiment 2 (every 10 iterations)

\subsubsection{Experimental analysis and conclusion}

In the above experiments, the atmospheric diffusion process of single and multiple sources is simulated. The experimental results are carried out in a closed grid space, which can be infinite in theory. Because the experiment is based on grid and has no relation with real physical quantity, it is suitable for diffusion process of various scales.

The meaning of 10 iterations in Fig. 4 and Fig. 5 refers to the process of 10 steps diffusion evolution of each image interval, that is, the diffusion time interval of each image is $1 \mathrm{~s}$. In the picture, the darker the color, the higher the concentration of pollutants. Figure 4 is the expression of the atmospheric diffusion process of a single release source. Because the wind speed in three directions is not equal, a regular elliptical diffusion surface is formed; Figure 5 is the expression of the diffusion process of a release source at two different positions and with different intensities. When the two sources are superposed, the pollutant concentration in the superposed area increases significantly. In addition, because this experiment uses the way of cellular automata to realize the diffusion process, the pollutant concentration in any time and any space can be calculated and expressed, which is very intuitive and analyzable. 


\subsection{Wind tunnel experiment}

The reliability of the method is verified by wind tunnel experiment. The equivalent release parameters of wind tunnel test are shown in Table 3. The range of experimental simulation is $15 \mathrm{~km} \times 15 \mathrm{~km} \times 1.15 \mathrm{~km}$. According to the measured meteorological data of the meteorological tower, the wind field corresponding to the actual situation is generated by setting appropriate rough elements on the bottom plate. The tracer gas is released from the release point. After the tracer gas concentration distribution is stable, the tracer gas concentration is measured.

\begin{tabular}{|c|c|c|c|}
\hline $\begin{array}{c}\text { Release } \\
\text { height }\end{array}$ & $\begin{array}{c}\text { Duration of } \\
\text { release }\end{array}$ & $\begin{array}{c}\text { wind } \\
\text { direction }\end{array}$ & $\begin{array}{c}\text { Atmospheric } \\
\text { stability }\end{array}$ \\
\hline $22 \mathrm{~m}$ & $4 \mathrm{~h}$ & West & $\mathrm{D}$ \\
\hline
\end{tabular}

Table 3. Design parameters of wind tunnel experiment

\subsubsection{Three-dimensional wind field}

According to the data in Table 3, the three-dimensional wind field needed for the simulation calculation of the diffusion model is generated. The grid in the horizontal direction of the wind field is $100 \mathrm{~m} \times 100 \mathrm{~m}$, and the vertical direction is divided into 21 layers. Fig. 6 (a) is the wind field of the second floor $(5.709 \mathrm{~m}$ high from the ground) at the time of 0 , and Fig. 6 (b) is the wind field of the second floor 1 hour after the release, where the red point is the location of the release source.

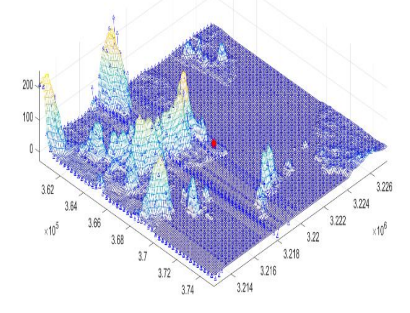

(a) the wind field of the second floor at the time of 0

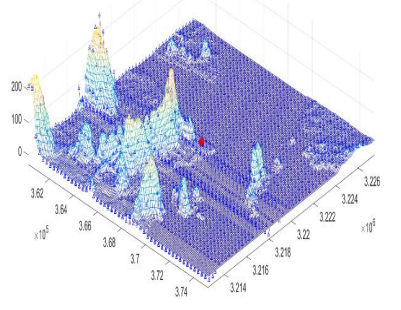

(b) the wind field of the second floor 1 hour after the release
Figure 6. Wind field at different times of the same height

Figure 7 (a) shows the wind field at 0 time of the third floor (17.128m high from the ground), and Figure 7 (b) shows the wind field at 0 time of the fifth floor $(39.965 \mathrm{~m}$ high from the ground), where the red point is the location of the release source.

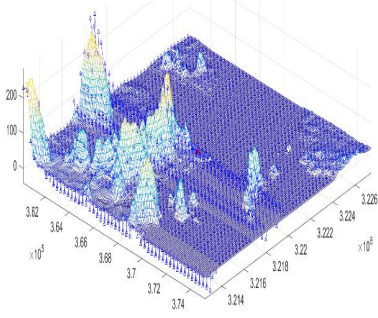

(a) the wind field of the third floor at the time of 0

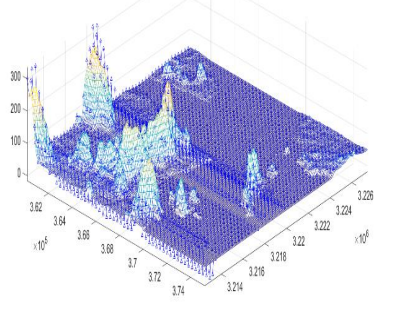

(b) the wind field of the fifth floor 1 hour after the release

Figure 7 . Wind fields of different heights at the same time

\subsubsection{Comparison of wind tunnel simulation results}

Under the same meteorological conditions and release conditions, the results of atmospheric pollution diffusion simulation calculation based on cellular automata are shown in Figure 8. Figure 8 (a), (b), (c) and (d) show the concentration distribution after 5 minutes, 15 minutes, 40 minutes and 60 minutes respectively. (c) concentration distribution after 40 minutes of release

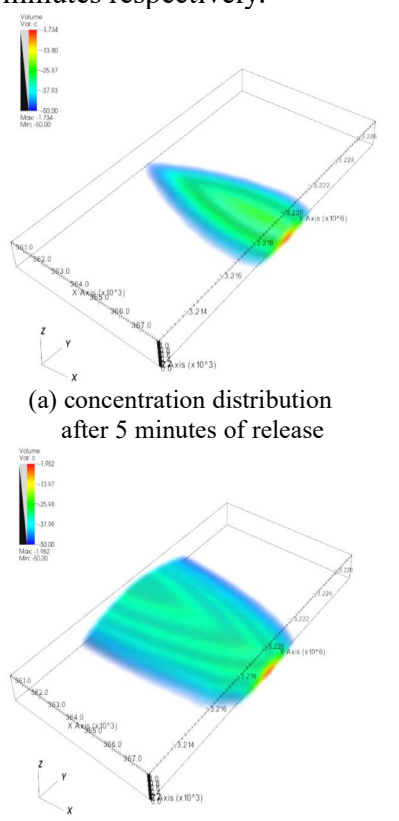

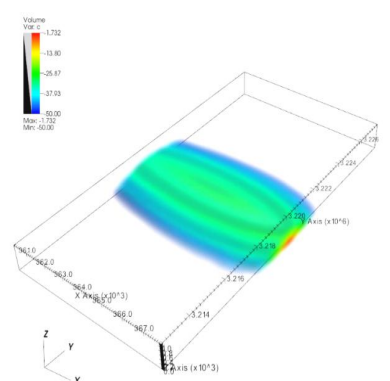

(b) concentration distribution after 15 minutes of release

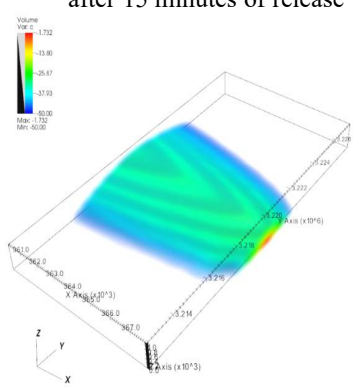

(d) concentration distribution after 60 minutes of release
Figure 8. Concentration distribution at different time

The calculation results after 4 hours of simulated release are compared with the wind tunnel test results. The comparison of smoke plume results is shown in Figure 9, and the distribution comparison of atmospheric dispersion factors at different observation points is shown in Figure 10.

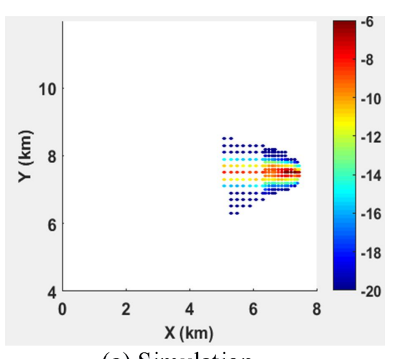

(a) Simulation

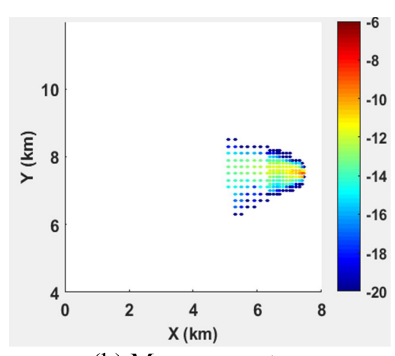

(b) Measurement
Figure 9. Comparison of plume results

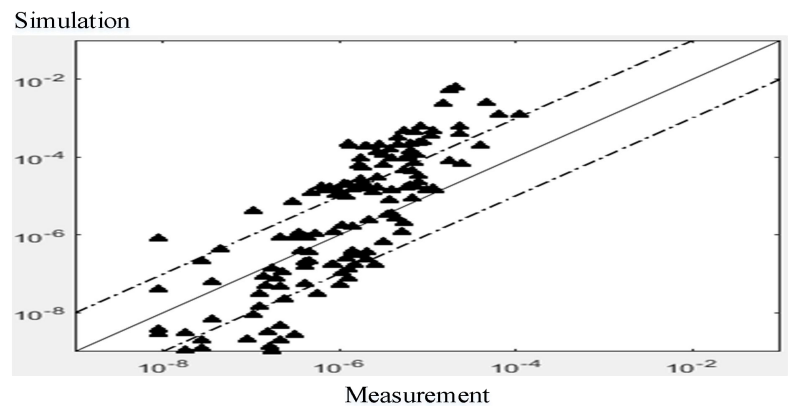

Figure10. Scatter diagram of atmospheric dispersion

factor

The comparison of statistical results is shown in Table 4, the 
first column is the statistical index; the second column is the statistical analysis of all 200 measuring points; the third column is the statistical analysis of the measuring points 19 , where the maximum measurement value of the axis is; the fourth column is the reference value of several parameters.

\begin{tabular}{|c|c|c|c|}
\hline Parameter & $\begin{array}{c}\text { All } \\
\text { measuring } \\
\text { points }\end{array}$ & $\begin{array}{c}\text { Maximum } \\
\text { measuring } \\
\text { point of axis }\end{array}$ & $\begin{array}{c}\text { Reference } \\
\text { range }\end{array}$ \\
\hline $\begin{array}{c}\text { Total number } \\
\text { Non-zero number }\end{array}$ & 200 & 19 & \\
FA2 & 0.10 & 19 & \\
FA5 & 0.31 & 0.16 & $0.5 \sim 2$ \\
MG & $7.65 \times 10^{-1}$ & $7.44 \times 10^{-2}$ & $0.2 \sim 5$ \\
VG & $1.33 \times 10^{5}$ & $2.37 \times 10^{3}$ & $1 \sim 1.6$ \\
\hline
\end{tabular}

Table 4. Statistical result chart

\subsubsection{Analysis of test results}

It can be seen from the test results that the plume is continuous and similar in shape, which can reflect the diffusion of wind tunnel experiments. However, the concentration of the ground axis is high and the edge of both sides of the plume is slightly low. The main reasons are as follows:

(1) Error of diffusion parameter calculation. There are some errors in the calculation method of diffusion parameters used in this paper. From the comparison between the simulation results and the actual results, it can be seen that the diffusion of y-axis is not enough, leading to a little narrow plume shape.

(2) Error caused by discretization. The diffusion process is continuous, but the calculation in this paper needs to discretize the diffusion region. The accuracy of the numerical solution has a great relationship with the size of the grid.

(3) Error of three-dimensional wind field calculation. The wind field calculated by the diagnostic method deviates from the real wind field.

(4) Error of spatial interpolation algorithm. The data of wind field is on the grid. The data (wind field data, diffusion parameters) on the eight grid points of the three-dimensional grid (cell) need to be calculated by spatial interpolation to get the data of the three-dimensional grid (cell). Because the spatial interpolation used in this paper is trilinear interpolation, the result of interpolation is wrong with the actual result, which leads to the difference between the simulation result and the actual result.

\section{CONCLUSION}

Based on the traditional spatial data model and the theory of solid grid and cellular automata, this paper proposes a new spatial data model which is a mixture of state-oriented modeling and object-oriented modeling. The model supports true threedimensional grid representation and cellular automata deduction, and can combine the calculation and expression of atmospheric pollution. This improves the calculation accuracy and expression efficiency.

In the actual atmospheric diffusion events, more complex factors are involved, which is exactly the advantage of the model. Data modeling based on state information is adopted, and all external factors are stored as grid state or attribute. Because the number of grids, time span and granularity are fixed, the data management will not be more difficult due to the increase of the number of external factors and the change of the number of pollution sources
The diffusion expression of this paper is obtained by discretization of Euler model. The model does not consider the factors such as pollutant source term, dry and wet deposition, topography and so on, and the accuracy of the model needs to be improved. However, the data model and the application method of cellular automata designed in this paper are theoretically applicable to all kinds of models.

In the future, we need to study the gridding process deduction expression method for the diffusion model of various kinds of atmospheric pollution. In addition, the cell method based on the three-dimensional grid has a large amount of computation, and the external environment is relatively stable in a certain area when there are many times. So it will be an important research topic to use the variable scale grid method to simulate the cellular automata.

\section{REFERENCES}

Raza, S. S., Iqbal, M., 2005. Atmospheric dispersion modeling for an accidental release from the Pakistan Research Reactor1(PARR-1). Annals of Nuclear Energy, 32(11), 1157-1166.

Thykier-Nielsen, S., Deme, S., Mikkelesn, T., 1999. Description of the atmospheric dispersion module RIMPUFF.

Yu, Q., Liu, Y.Z., 2000. Application of Lagrangian puff model in the early stage of a nuclear emergency. Journal of Tsinghua University (Science and Technology), 40(12), 6-9.

Yan, Z., Yang, Y. X., Zhang, Y., 2009. Monte-Carlo simulation of early plume after nuclear accident. Journal of East China Institute of Technology (Natural Science), 32(4), 358-361.

Zhang, M. G., Han, Z. W., Lei, X. E., 2009. A numerical study of atmospheric dispersion in the Area of nuclear power plant Dayawan. Climatic and Environmental Research, 5(1), 90-95.

Lauritzen, B., Baklanov, A., Mahura, A., 2006. K-model description of probabilistic long-range atmospheric transport in the Northern Hemisphere. Atmospheric Environment, 40(23), 4352-4369.

Lagzi, I., Karman, D., Turanyi, T., et a1, 2004. Simulation of the dispersion of nuclear contamination using an adaptive Eulerian grid model. Journal of Environmental Radioactivity, 75(1), 59-82.

de Sampaio, P. A. B., Junior, M. A. G., Lapa, C. M. F., 2008. A CFD approach to the atmospheric dispersion of radionuclides in the vicinity of NPPs. Nuclear Engineering and Design, 238(1), 250-273.

$\mathrm{Xu}$ B. L., 2009. A Prototype of Collaborative Virtual Geographic Environments to Facilitate Air Pollution Simulation. Hong Kong: CUHK, 1-260.

Wang, Y. X., Sun, Z. H., Zhang, P. P., et al, 2011. Research on the visualization representation method of air quality simulation result based on GIS. Geography and Geo-Information Science, $27(2), 46-50$

Sun, X. Z., Dong, X. M., Ren, Z. B., 2011. Visualization of atmospheric point source dispersion model based on GIS. Science of Surveying and Mapping, 36(1), 24-26.

Chen, W. J., Chen, S. Z., Du, E. E., et al, 2011. Design and development of emergency monitoring system to sudden air 
pollution accidents. Journal of Geo-Information Science, 13(2), $65-72$.

Zhang, P. P., Wang, Y. X., Wu, Q. Y., et al, 2010. Integration and application of GIS and atmospheric environment model. Research of Environmental Sciences, 23(5), 575-580.

Vienneau, D., Hoogh, K., Briggs, D., 2009. A GIS-based method for modelling air pollution exposures across Europe. Science of the Total Environment, 408(2), 255-266.

Theophanides, M., Anastassopoulou, J., 2009. Air pollution simulation and geographical information systems(GIS) applied to Athens International Airport. Journal of Environmental Science and Health Part A, 44(8), 758-766.

Elbir, T., 2004 A GIS based decision support system for estimation, visualization and analysis of air pollution for large Turkish cities. Atmospheric Environment, 38(27), 4509-4517.

Li, H. W., Zhang, S. Q., Sun, Q. Z., 2007. Research on visualization of point-pollution source diffuseness simulation. Environmental Science and Management, 32(10), 175-178.

Hu, Y., Zhu, J., Lin H., et al, 2013. A study on multidimensional dynamic visualization of air pollution based on atmospheric pollution dispersion model. Chinese High Technology Letters, 23(7),728-734.

Zhu, Q., 2004. A survey of three dimensional GIS technologies. Geomatics World, 03, 8-12.

Lai, G. L., Tong, X. C., Ding, L., et al, 2018. Multiscale Integer Coding and Data Index of 3DSpatial Grid. Acta Geodaetica et Cartographica Sinica, 47(7), 1007-1017.

Orenstein, J. A., Merrett, T. H., 1984. A Class of Data Structures for associative Searching. Proceedings of the 3rd ACMSIGACTSIGMOD Symposium on Principles of Database Systems, 181-190.

Chen, G.H., Huang, T. F., Chen, W. J., Jia, M. S., 2013. Dynamic simulation system of leakage and diffusion of hazardous chemical gas based on real 3D GIS. Journal of Safety Science and Technology, 1(1), 113-118. 